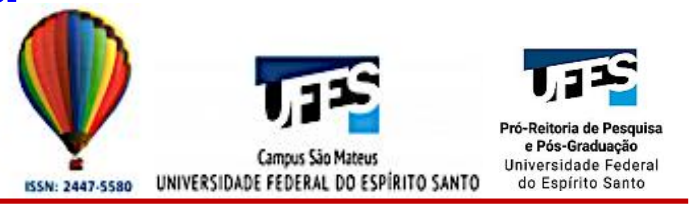

ARTIGO ORIGINAL

OPEN ACCESS

\title{
BUSINESS INTELLIGENCE COMO SUPORTE À TOMADA DE DECISÃO: O ESTADO DA ARTE POR MEIO DO PROKNOW-C
}

\author{
BUSINESS INTELLIGENCE AS A SUPPORT FOR DECISION MAKING: THE STATE OF THE ART \\ THROUGH PROKNOW-C
}
Marco Antônio Albertino Schinaider ${ }^{1}$, Viviane Nunes Tetzlaff Lee ${ }^{2}$, \& Marcos Wagner Jesus Servare Junior ${ }^{3 *}$
123 Centro Universitário Salesiano - UniSales.
${ }^{1}$ marcoalbertino@outlook.com ${ }^{2}$ viviane.lee@salesiano.br ${ }^{3 *}$ marcos.servare@ salesiano.br

\section{ARTIGO INFO.}

\section{Recebido em: 29.11.2021}

Aprovado em: 02.02.2022

Disponibilizado em: 08.02.2022

\section{Palavras-chave:}

Business intelligence; Tomada de decisão; estratégia; Proknow-C.

\section{KEYWORDS:}

Business intelligence; Decision making; strategy; Proknow-C.

*Autor Correspondente: Servare, M. W. J., Jr.

\section{RESUMO}

A sociedade nunca deixou de ser afetada por transformações impulsionadas pelas revoluções ao longo do tempo, e a forma do manuseio dos dados nas organizações faz parte destas transformações, a refletir diretamente na assertividade das decisões tomadas. Por este ângulo, o presente artigo abordará o estado da arte sobre a metodologia business intelligence $(B I)$ na tomada de decisão. O Objetivo geral compreende elucidar a relevância da implementação de $B I$ como alicerce na tomada de decisão estratégica. Para alcançar o objetivo será realizada uma pesquisa bibliográfica, aplicando o método de formação do portfólio bibliográfico e análise bibliométrica, Proknow-C. A pesquisa resultou em 3646 artigos. Ficou evidente que as organizações devem avaliar o sistema mais adequado as suas necessidades de informação a respeitar o orçamento estipulado dentro da etapa de validação do projeto, pois o alto custo, além da complexidade dos sistemas, são fatores que atrapalham a implantação. O sucesso competitivo é atrelado ao ambiente de decisão que o usuário está inserido. Quanto mais complexo for o ambiente e mais variáveis atingirem o indivíduo, maior a dificuldade de acesso à informação ideal para tomada de decisão.
Em suma, a pesquisa atingiu o seu objetivo de elucidar os impactos do $B I$ nas empresas como provedor do manuseio estratégico de informações baseado nas métricas relacionadas ao planejamento estratégico para auxílio na tomada de decisão.

\begin{abstract}
Society has never ceased to be affected by transformations driven by revolutions over time, and the way data is handled in organizations is part of these transformations, reflecting directly on the assertiveness of the decisions taken. From this angle, this article will address the state of the art on business intelligence (BI) methodology in decision making. The general objective comprises elucidating the relevance of implementing BI as a foundation in strategic decision making. To achieve the objective, a bibliographic research will be carried out, applying the method of formation of the bibliographic portfolio and bibliometric analysis, Proknow-C. The search resulted in 3646 articles. It was evident that organizations must evaluate the most appropriate system for their information needs, respecting the budget stipulated within the project validation stage, since the high cost, in addition to the complexity of the systems, are factors that hinder the implementation. Competitive success is linked to the decision environment in which the user is inserted. The more complex the environment and the more variables that affect the individual, the greater the difficulty in accessing the ideal information for decision making. In short, the research achieved its objective of elucidating the impacts of BI on companies as a provider of strategic handling of information based on metrics related to strategic planning to aid in decision making.
\end{abstract}


Citação (APA): Schinaider, M. A. A., Lee, V. N. T., \& Servare, M. W. J., Jr. (2022). Business intelligence como suporte à tomada de decisão: o estado da arte por meio do Proknow. Brazilian Journal of Production Engineering, 8(2), 79-98.

\section{INTRODUÇÃ̃O}

Os dizeres: "um mundo de constante mudanças", apesar de trivial, nunca fez tanto sentido como na era contemporânea. Desde o surgimento da primeira forma de vida, o primeiro ser complexo, a sociedade nunca deixou de ser afetada por transformações impulsionadas pelas revoluções ao longo do tempo, mas nada se compara a atual revolução 4.0, que tem modificado o ambiente empresarial através da tecnologia da informação (TI).

A forma do manuseio dos dados nas organizações faz parte disto, eles estão surgindo de novas origens e são utilizados em diversos setores das organizações por uma perspectiva mais dinâmica se comparado a tempos antes, em que a utilização se dava isoladamente setor a setor. A nova visão pauta-se em como os dados podem ser atravessados entre si e observar como se comportam (Rogers, 2017). As Informações geradas precisam ser manuseados de forma estratégica.

No passado a leitura dos dados era mais difícil, apesar da grande quantidade, não havia suporte na capacidade de processamento como nos tempos atuais. Os saltos dados pela TI resultaram em tecnologias capazes de processar uma gigantesca quantidade de informação com eficiência. O sucesso no trabalho com dados consiste no uso adequado, com o suporte de ferramentas necessárias para gerar informação e fazer verificações preditivas (Armstrong, 2019). Assim, as inovações na TI avançam de modo similar em todas as áreas. Numa abordagem que exemplifica esta afirmativa, Schwab (2016) relata que tanto o sequenciamento genético, quanto a tecnologia robótica dependem da capacidade de processamento para avançar.

Neste contexto, fica evidente a importância que estruturas correlacionadas ao manuseio de dados tem dentro do ambiente organizacional a contribuir para o sucesso competitivo. Por este ângulo, o presente artigo abordará o estado da arte sobre business intelligence (BI) na tomada de decisão. O objetivo geral compreende elucidar a relevância da implementação de $B I$ como alicerce na tomada de decisão estratégica. Para isto, esta pesquisa contará com três objetivos específicos, procurar na literatura se $B I$ atua como agente de promoção de competitividade empresarial por meio da tomada de decisão e qual o escopo necessário à sua implementação, entender o alinhamento entre os resultados obtidos com o uso de $B I$ e os parâmetros referenciais no plano corporativo e constatar se $B I$ tem recebido importância na área acadêmica por meio do debate em estudos.

A pesquisa tem por objetivo geração de aprendizado a procurar responder dilemas, é parte que compõe a ciência, da qual busca-se extrair a verdade (Zanella, 2009). Nesta pesquisa será tomada a ordem de classificação de análise proposta por Vergara (2016), que determina os tipos de pesquisas quanto aos fins e aos meios.

Quanto aos fins esta pesquisa é tipificada como exploratória, explicativa e metodológica. É classificada como exploratória por se tratar de um tema ainda pouco explorado, qual seja "business intelligence na gestão organizacional". É explicativa pois procura compreender e explicar fatores ligados a competitividade, estratégia corporativa e implementação de ferramentas, que fazem do $B I$ sucesso ou insucesso nas empresas. E classifica-se ainda como metodológica por utiliza-se de um procedimento metodológico construtivista para a formação de portfólio bibliográfico, e que atenderá o problema de pesquisa. 

decisão: o estado da arte por meio do Proknow. Brazilian Journal of Production Engineering, 8(2), 79-98.

No que tange aos meios esta pesquisa é definida como pesquisa bibliográfica por pautar o estudo em artigos selecionados criteriosamente em bases de periódicos conceituados dispostos no portal da Coordenação de Aperfeiçoamento de Pessoal de Nível Superior (CAPES). Tratase da pesquisa entre os anos 2010 a 2020.

A importância deste estudo está na necessidade do processo de atualização constante nas empresas, a implantar métodos e ferramentas que possam contribuir com o aumento da performance organizacional. É esperado que esta pesquisa ajude as empresas a desenvolverem cada vez mais interesse pelo assunto do artigo e gere resultados a possibilitar implementação e adoção de estruturas de BI mais assertivas, a solução deste estudo poderá ser compartilhada entre empresas e em projetos futuros nesta área, e também contribuir com a sociedade acadêmica.

A abordagem do conceito de $B I$ neste artigo não se restringirá somente como sistema.

Nedelcu (2013) relata que a expressão business intelligence é datado há mais tempo, do que o surgimento da necessidade de sua aplicação em relatórios empresariais, o termo surgiu na década de 90 pelo Gartner Group. Ainda assim, seu crescimento se deu pela necessidade de as empresas analisarem as informações advindas da grande gama de dados. A vantagem do uso de BI está no aproveitamento de aspectos novos e antigos, que muito contribuem com a organização. Na visão defendida por Agiu, Mateescu $e$ Muntean (2014), BI é definido como um conjunto de ferramentas, como softwares, aplicativos, ou outras formas que auxiliam a organização no processo de tomada de decisão, por meio da análise de dados. Este método aplicado nas empresas promove melhorias no relacionamento com os stakeholders.

Delen, Turban e Sharda (2014) conceituam BI por uma noção mais ampla, segundos os autores o foco consiste na interação dos dados para que os gestores sejam capazes de tomar decisões assertivas e performarem melhor.

Para Silva, Bezerra, Rios e Amorim (2018), BI originou-se pela necessidade de administrar uma grande quantidade de dados que surgem em empresas de todos os portes e que são ineficazes quando mal geridos. Abusweilem and Abualoush (2019) corroboram ressaltando que BI promove a visão holística sobre o negócio, com conhecimento assertivo advindo de dentro e fora da organização. Esse conhecimento é resultado dos dados acumulados da organização nos três níveis empresariais: operacionais, táticos e estratégicos.

A finalidade deste estudo será responder a seguinte indagação: quais os impactos do BI nas empresas como provedor de manuseio estratégico de informações baseado nas métricas relacionadas ao planejamento estratégico para auxilio na tomada de decisão?

A pesquisa será aplicada em bases de artigos da CAPES aplicando o método de formação do portfólio bibliográfico e análise bibliométrica, Knowledge Development Process Constructivist (ProKnow-C) método aplicado em estudos de diversas áreas (Afonso, Souza, Ensslin, \& Ensslin, 2011; Ensslin, et al., 2014; Vaz, Tasca, Ensslin, Ensslin, \& Selig, 2012; Lizot, Junior, Magacho, Bortoluzzi, \& Viero, 2016; Santos, Schenatto, \& Oliveira, 2017; Vieira, Bortoluzzi, Costa, \& Lima, 2017; Lacerda, Ensslin, \& Ensslin, 2012; Reina, Ensslin, Ensslin, \& Reina, 2014). 

decisão: o estado da arte por meio do Proknow. Brazilian Journal of Production Engineering, 8(2), 79-98.

A escolha pela metodologia Proknow-C consiste na necessidade da pesquisa bibliográfica apoiar-se em um processo validado para junção do material de estudo com credibilidade. Afonso e outros (2011) ressaltam esta questão, aplicar uma revisão de literatura sem um método conceituado pode atrapalhar o autor a manter o foco por uma única trilha a ser seguida e dedicar o tempo a obras não tão proveitosas ao estudo em detrimento de obras importantes. Outro ponto é que a grande disponibilidade de estudos pode confundir o autor.

Após montagem do portfólio bibliográfico será aplicada a análise sobre o material selecionado com bases nos objetivos supracitados.

\section{PROKNOW-C}

Este trabalho de pesquisa conforme mencionado abordou o processo metodológico Proknow-C para formação do portfólio bibliográfico, alvo de análise. A metodologia Proknow-C é relatada em muitos estudos como ferramenta para formação de portfólio bibliográfico de forma embasada e criteriosa, por meio de um processo construtivista (Ensslin et al., 2014; Santos et. al., 2017; Vieira et al., 2017; Lacerda et al., 2012). Esse método é composto por quatro etapas: (I) seleção do portfólio bibliográfico, (II) análise bibliométrica, (III) análise sistêmica e (IV) oportunidades de pesquisa (Reina et al., 2014). Para este estudo foram adotadas as etapas portfólio bibliográfico, análise bibliométrica e análise sistêmica.

Por se tratar de um processo construtivista é levado em conta a percepção do autor sobre o tema para execução do fluxo de formação do portfólio bibliográfico: formação de palavras-chaves, escolha da base de dados, alinhamento do título, alinhamento do resumo e disponibilização do artigo na íntegra (Fontana, 2020).

Visando um melhor entendimento a respeito da aplicação da metodologia Proknow-C em produções científicas, abaixo são elencadas pesquisas que obtiveram resultados utilizando a metodologia para a formação de portfólio bibliográfico:

a) AUTORES E ANO: Afonso et al. (2011);

TEMA: avaliação de desempenho da sustentabilidade;

TAMANHO DO PORTFÓLIO BIBLIOGRÁFICO: 13 artigos;

RESULTADO: amostra de artigos embrionária comparado aos ramos tradicionais da ciência.

b) AUTORES E ANO: Ensslin et al. (2014);

TEMA: avaliação de desempenho de processos de implementação de eficiência energética

TAMANHO DO PORTFÓLIO BIBLIOGRÁFICO: 13 artigos.

RESULTADO: a área do tema configura-se como um campo a ser explorado.

c) AUTORES E ANO: Vaz et al. (2012);

TEMA: avaliação de desempenho na gestão estratégica organizacional;

TAMANHO DO PORTFÓLIO BIBLIOGRÁFICO: 12 artigos;

RESULTADO: construção de conhecimento sobre o tema pelos pesquisadores e identificação de lacunas.

d) AUTORES E ANO: Lizot et al. (2016);

TEMA: avaliação de desempenho na gestão da produção;

TAMANHO DO PORTFÓLIO BIBLIOGRÁFICO: 11 artigos;

RESULTADO: dissertação de análise descritiva pautada em seis lentes propostas pelos autores com base na literatura. 

decisão: o estado da arte por meio do Proknow. Brazilian Journal of Production Engineering, 8(2), 79-98.

e) AUTORES E ANO: Santos et al. (2017);

TEMA: previsão de demanda utilizando séries temporais;

TAMANHO DO PORTFÓLIO BIBLIOGRÁFICO: 24 artigos;

RESULTADO: área da pesquisa já é bastante explorada o que dificultou a identificação de lacunas na literatura.

f) AUTORES E ANO: Vieira et al. (2017);

TEMA: avaliação do nível de maturidade das empresas na utilização de ferramentas Lean Manufacturing;

TAMANHO DO PORTFÓLIO BIBLIOGRÁFICO: 24 artigos;

RESULTADO: construção de conhecimento sobre o tema pelos pesquisadores e identificação de lacunas.

g) AUTORES E ANO: Lacerda et al. (2012);

TEMA: gerenciamento do portfólio de projetos;

TAMANHO DO PORTFÓLIO BIBLIOGRÁFICO: 8 artigos;

RESULTADO: o conhecimento gerado permitiu medir cardinalmente o nível de contribuição que um projeto possui para a meta geral.

h) AUTORES E ANO: Reina et al. (2014);

TEMA: seleção de projetos;

TAMANHO DO PORTFÓLIO BIBLIOGRÁFICO: 23 artigos;

RESULTADO: construção de conhecimento sobre o tema pelos pesquisadores e identificação de lacunas.

\subsection{Seleção de portfólio bibliográfico}

\subsubsection{Definição de palavras-chaves}

A princípio foram elencados os eixos de pesquisa e então foram formuladas as palavras-chaves (Vaz et al., 2012). O eixo de pesquisa deve ser descrito com base no tema, assim, para este artigo foram definidos eixos de acordo com o tema "business intelligence na gestão organizacional". As palavras-chaves derivaram do eixo de pesquisa e foram traduzidas para língua inglesa, visando alcançar o maior número de artigos nas bases internacionais. Para este estudo o eixo de pesquisa e as palavras-chaves se deram conforme o Quadro 1.

Quadro 1. Eixo de pesquisa e palavras-chaves.

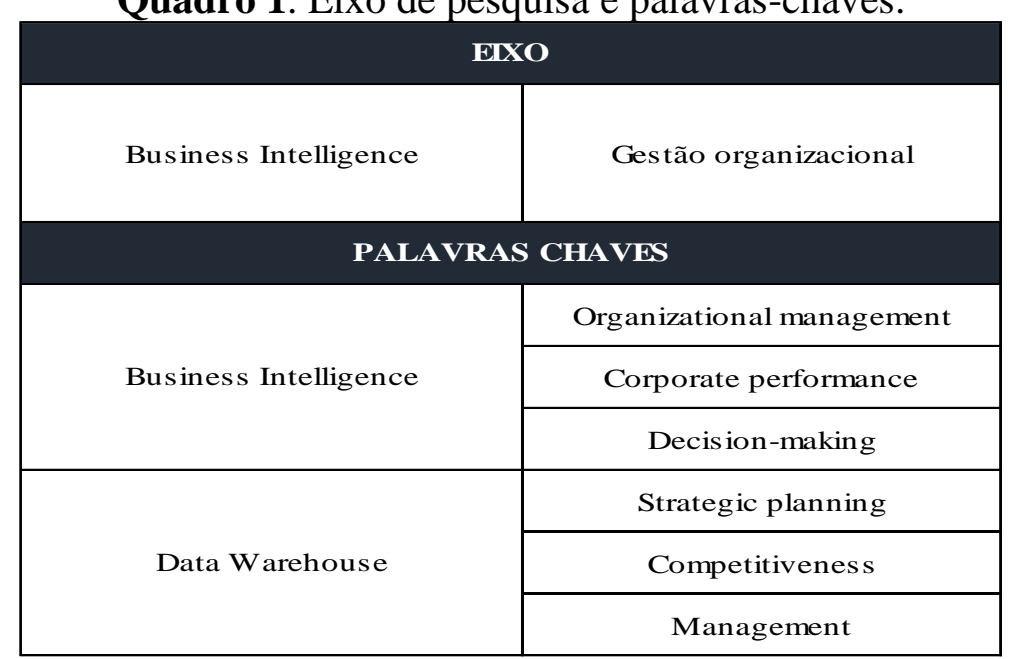

Fonte: Autores (2021).

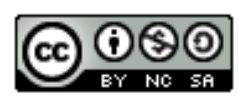



decisão: o estado da arte por meio do Proknow. Brazilian Journal of Production Engineering, 8(2), 79-98.

Foi montada uma expressão booleana para a busca nas bases visando atingir o melhor aproveitamento entre a combinação das palavras-chaves: ("business intelligence" OR "data warehouse") AND ("organizational management" OR "corporate performance" OR "decisionmaking" OR "strategic planning" OR competiti** OR manag**).

\subsubsection{Definição do banco de dados}

As bases escolhidas para o estudo foram a Scielo, a Scopus e a Web of Science (WOS). Em todas as bases a pesquisa se limitou a "artigo" e no período que compreende o ano de 2010 ao ano de 2020, a busca de artigos se deu no mês de setembro de 2020. Foram escolhidos dois artigos mais próximos ao tema para comparação e alinhamento das palavras-chaves (Lizot et al., 2016), os artigos escolhidos foram:

a) Alasiri, Salameh. (2020) The impact of business intelligence (BI) and decision support systems (DSS): exploratory study. International Journal of Management-IJM, 11(5), 10011016. https://doi.org/10.34218/IJM.11.5.2020.087.

Palavras chaves: BI, DSS, Business Intelligent, Decision Support System, Organizational Strategy.

b) Vaish, Shrivastava, Sen. (2020) Business intelligence: escalation of data warehousing and data mining for effective decision making. International Journal of Advanced Science and Technology, 29(5), 1377-1388. http://sersc.org/journals/index.php/IJAST/article/view/8178. Palavras chaves: Data Warehouse, Data Mining, Business Intelligence, Internet of Things, Artificial Intelligence, Decision Support System.

Dessa forma, foi acrescentada ao quadro de palavras-chave as palavras data mining, relacionada ao eixo business intelligence, que a princípio não havia sido pensada para compor a expressão de busca. A pesquisa foi refeita incorporando a nova palavra chave.

A pesquisa resultou em 3.646 artigos estando dentro da quantidade ideal para aplicação do método, de acordo com Lacerda et al. (2012), a pesquisa de artigos brutos deve retornar de 1.000 a 12.000 artigos. Os artigos vieram divididos entre as bases conforme o Gráfico 1.

Gráfico 1. Quantidade de artigos por base.

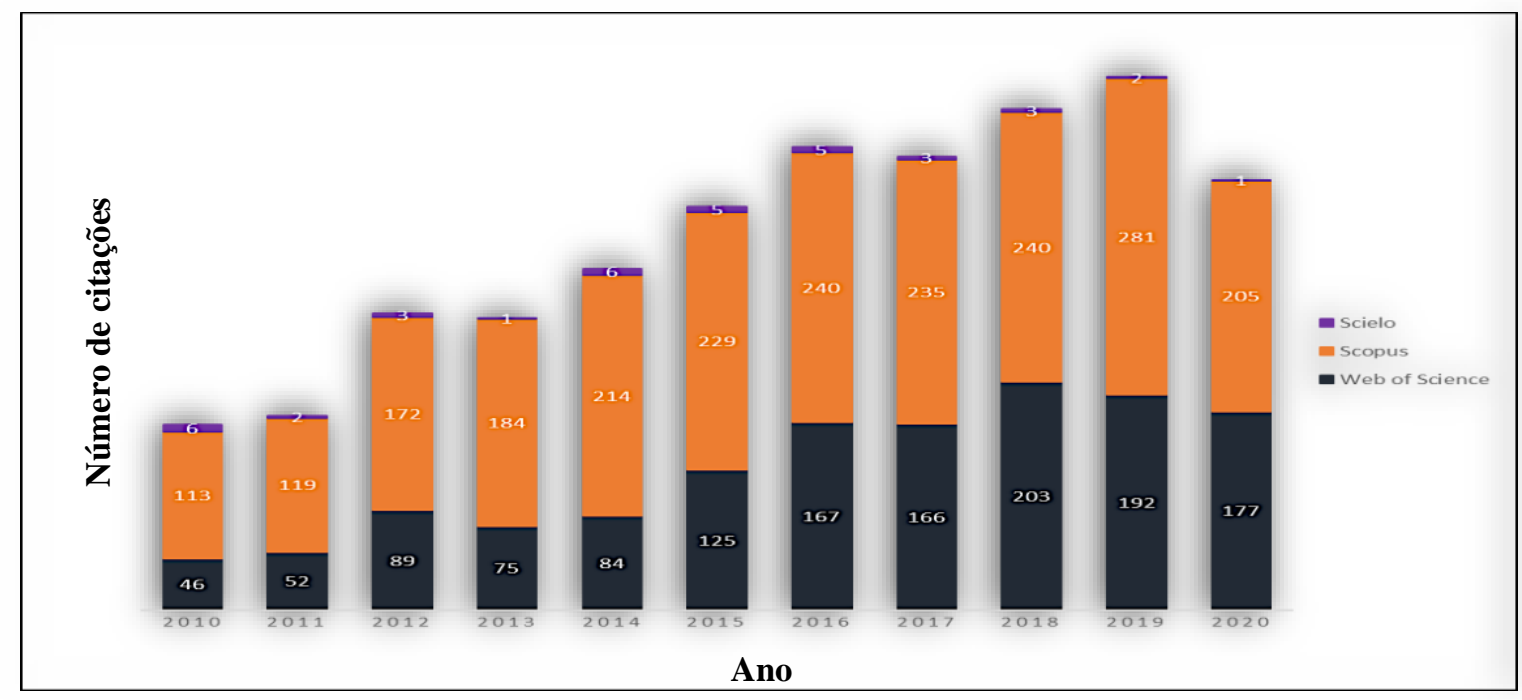

Fonte: Autores (2021).

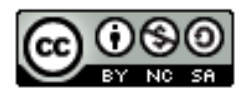



decisão: o estado da arte por meio do Proknow. Brazilian Journal of Production Engineering, 8(2), 79-98.

Conforme percebido há uma tendência de crescimento do número das publicações sobre business intelligence ao longo dos anos, se comparado ao número de publicações no ano de 2010, ano mais distante dentro da linha temporal da pesquisa, e o ano de 2020 até o mês de setembro, quando foi realizado a busca nas bases, o crescimento do portfólio bruto ultrapassou $40 \%$, o que mostra maior interesse acadêmico dado ao assunto.

Na Figura 1 pode-se observar a nuvem de palavras que identifica os termos chaves mais citados pelos autores nos artigos, ficando em evidência as ligações em torno das palavras centrais do tema do artigo: business intelligence e management, mostrando a congruência entre os artigos retornados nas bases, mesmo antes do polimento.

Figura 1. Nuvem de palavras chaves artigos brutos. Fonte: autores

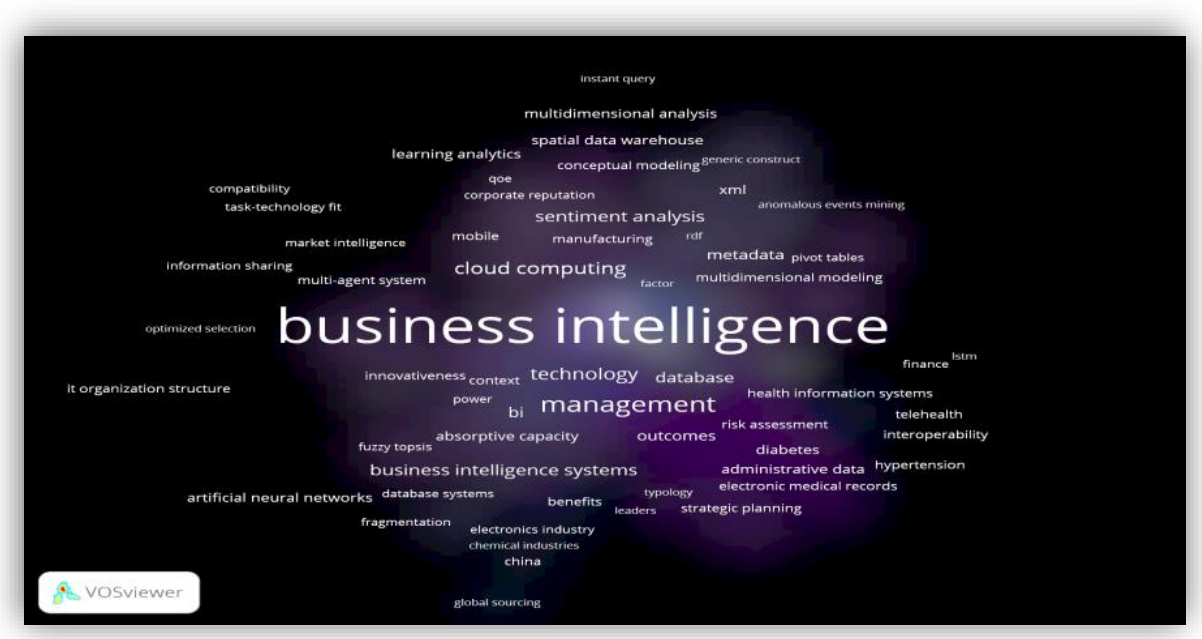

Fonte: Autores (2021).

\subsubsection{Filtragens do Banco de Artigos}

1) A filtragem de artigos brutos começou pela remoção dos arquivos duplicados e alinhamento do título ao tema de pesquisa (Vieira et al., 2017). Esta etapa resultou em 264 artigos.

2) O próximo passo foi sondar o número de citações dos artigos não duplicados e com títulos alinhados, no google scholar, conforme procedimento padrão do Proknow-C (Ensslin, Graziano, Dutra, \& Dezem, 2017). Para este estudo foi estabelecido a nota de corte de $80 \%$ sobre a representatividade dos artigos, resultando em 51 artigos, desta forma, a soma do número de citações dos 51 artigos selecionados equivale a $80 \%$ da soma total de citações dos artigos. Os artigos selecionados formaram o repositório $\mathrm{K}$.

3) Seguindo, foi analisado o resumo dos artigos do repositório $\mathrm{K}$ e eliminados 18 artigos que não estavam alinhados ao tema, restando 33 artigos que passaram a compor o repositório A.

4) Os artigos não selecionados na etapa anterior, ou seja, que tem o título alinhado ao tema, não duplicados, mas não reconhecidos cientificamente formaram o repositório $\mathrm{P}$ e passaram pela repescagem com 213 artigos. Duas ações são aplicadas, segundo a metodologia Proknow$C$ (Afonso et al., 2011):

a. Análise da data de publicação verificando se o artigo é recente - são considerados recentes os artigos com menos de dois anos de publicação.

b. Verificação se há autores que se repetem do repositório $K$.

5) Aplicado as regras acima chegou-se a 65 artigos que foram analisados os resumos, afunilando para 32 artigos com resumos alinhados e que passaram a compor o repositório B.

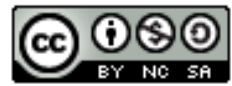

Esta obra está licenciada com uma Licença Creative Commons Atribuição-Não Comercial-Compartilha Igual 4.0 Internacional. Brazilian Journal of Production Engineering, São Mateus, Editora UFES/CEUNES/DETEC. 
Citação (APA): Schinaider, M. A. A., Lee, V. N. T., \& Servare, M. W. J., Jr. (2022). Business intelligence como suporte à tomada de decisão: o estado da arte por meio do Proknow. Brazilian Journal of Production Engineering, 8(2), 79-98.

6) Os repositórios A e B passaram a formar o repositório $\mathrm{C}$, o qual passou pela próxima etapa que consistiu na leitura da integra dos artigos e eliminação dos não alinhados ao tema ou não disponíveis na integra. No gráfico 2 é possível observar a quantidade de artigos em cada etapa da filtragem para formação do portfólio.

Gráfico 2. Quantidade de artigos por etapa

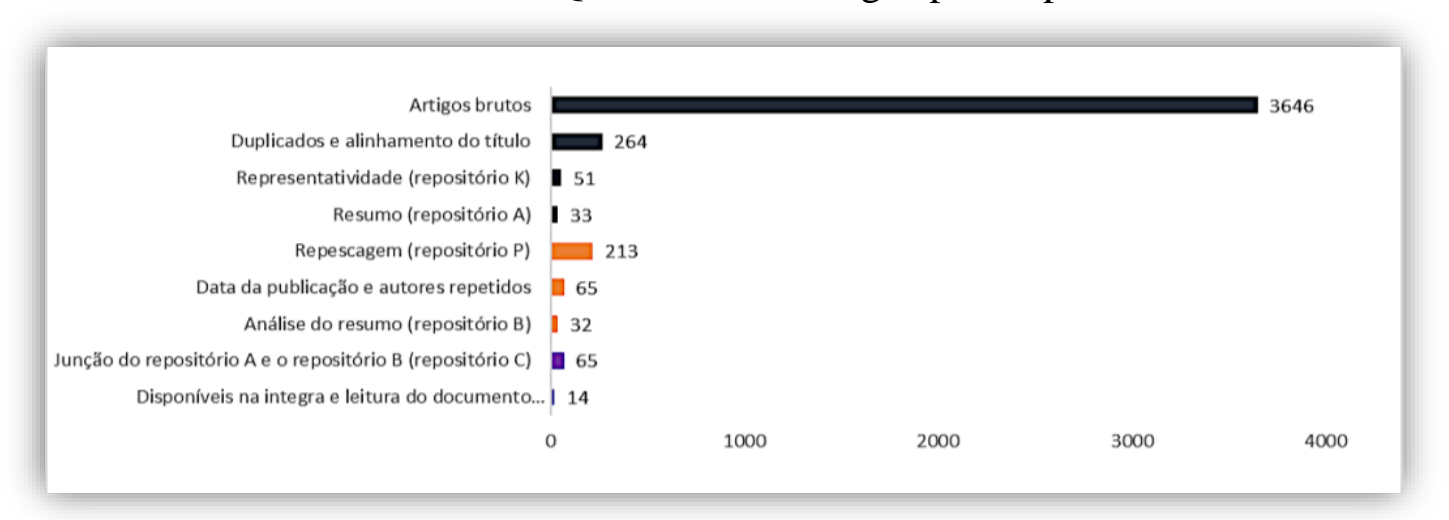

Fonte: Autores (2021).

A etapa final resultou em 14 artigos, que passaram a forma o repositório definitivo deste estudo, conforme relacionado abaixo no Quadro 2:

Quadro 2. Portfólio Selecionado.

\begin{tabular}{|c|c|c|c|}
\hline Título & Autor(es) & Ano & Número de Citações \\
\hline $\begin{array}{l}\text { Business intelligence: Escalation of data warehousing and } \\
\text { data mining for effective decision making }\end{array}$ & $\begin{array}{l}\text { Vaish, Shrivastava e } \\
\text { Sem. }\end{array}$ & 2020 & 0 \\
\hline $\begin{array}{c}\text { Identifying the main factors involved in business intelligence } \\
\text { implementation in SMEs }\end{array}$ & $\begin{array}{l}\text { Godinez, Coloapa, } \\
\text { Márquez, Mejía e } \\
\text { Gonzaga. }\end{array}$ & 2020 & 1 \\
\hline $\begin{array}{l}\text { The impact of business intelligence (BI) and decision } \\
\text { support systems (DSS): Exploratory study }\end{array}$ & Alasiri e Salameh. & 2020 & 0 \\
\hline $\begin{array}{l}\text { Role of business intelligence and analytics: Analysis of data } \\
\text { driven decision }\end{array}$ & Awasthi e Pandita. & 2019 & 0 \\
\hline Improving decision quality: The role of business intelligence & $\begin{array}{l}\text { Visinescu, Jones e } \\
\text { Sidorova. }\end{array}$ & 2017 & 42 \\
\hline $\begin{array}{l}\text { Extending the understanding of critical success factors for } \\
\text { implementing business intelligence systems }\end{array}$ & Yeoh e Popovic. & 2016 & 150 \\
\hline $\begin{array}{l}\text { Suboptimal business intelligence implementations: } \\
\text { Understanding and addressing the problems }\end{array}$ & $\begin{array}{l}\text { Boyton, Ayscough, } \\
\text { Kaveri e Chiong. }\end{array}$ & 2015 & 30 \\
\hline $\begin{array}{c}\text { Conceptualization of the business intelligence extended use } \\
\text { model }\end{array}$ & $\begin{array}{l}\text { Grubljesic e Jurij } \\
\text { Jaklic. }\end{array}$ & 2015 & 46 \\
\hline $\begin{array}{c}\text { Business intelligence adoption: A case study in the retail } \\
\text { chain }\end{array}$ & Olexová. & 2014 & 33 \\
\hline $\begin{array}{c}\text { Business intelligence success: The roles of BI capabilities } \\
\text { and decision environments }\end{array}$ & Isik, Jones e Sidorova. & 2013 & 413 \\
\hline $\begin{array}{c}\text { Information architecture analysis using business } \\
\text { intelligence tools based on the information needs of } \\
\text { executives }\end{array}$ & Affeldt e Junior. & 2013 & 26 \\
\hline $\begin{array}{l}\text { Towards business intelligence systems success: Effects of } \\
\text { maturity and culture on analytical decision making }\end{array}$ & $\begin{array}{l}\text { Popovic, Hackney, } \\
\text { Coelho e Jaklic. }\end{array}$ & 2012 & 400 \\
\hline $\begin{array}{c}\text { Critical success factors for implementing business } \\
\text { intelligence systems in small and medium enterprises on the } \\
\text { example of upper Silesia, Poland }\end{array}$ & Olszak e Ziemba. & 2012 & 240 \\
\hline Realizing the strategic impact of business intelligence tools & Sharma e Djiaw. & 2011 & 66 \\
\hline
\end{tabular}

$$
\text { Fonte: Autores (2021). }
$$

\subsection{Análise do Portfólio Bibliográfico}

Para conhecer as características do portfólio bibliográfico e entender a sua relevância na contribuição da pesquisa, são feitas análises conforme a seguir:

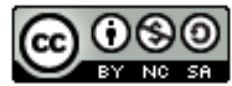

Esta obra está licenciada com uma Licença Creative Commons Atribuição-Não Comercial-Compartilha Igual 4.0 Internacional. Brazilian Journal of Production Engineering, São Mateus, Editora UFES/CEUNES/DETEC. 

decisão: o estado da arte por meio do Proknow. Brazilian Journal of Production Engineering, 8(2), 79-98.

- Relevância dos artigos: o entendimento da relevância dos artigos para a sociedade acadêmica é feito através do levantamento do número de citações em outros trabalhos acadêmicos utilizando o google scholar, é percebido que os artigos de maior relevância são: "Business intelligence success: The roles of BI capabilities and decision environments" $\mathrm{e}$ "Towards business intelligence systems success: Effects of maturity and culture on analytical decision making".

Gráfico 3. Artigos relevantes.

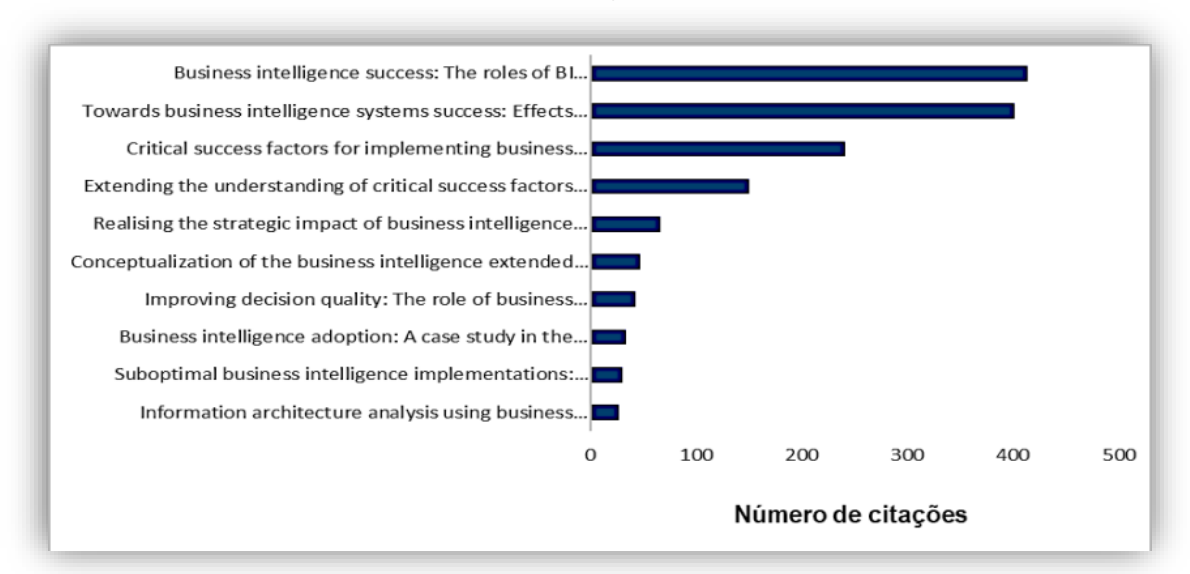

Fonte: Autores (2021).

- Relevância dos autores dentro do portfólio: no gráfico 4 é elencado o número de artigos que cada autor possui dentro do portfólio, é percebido que os autores que estão em dois artigos do portfólio são: Sidorova, A., Jaklic, J e Popovic, A.

Gráfico 4. Quantidade de artigos por autor.

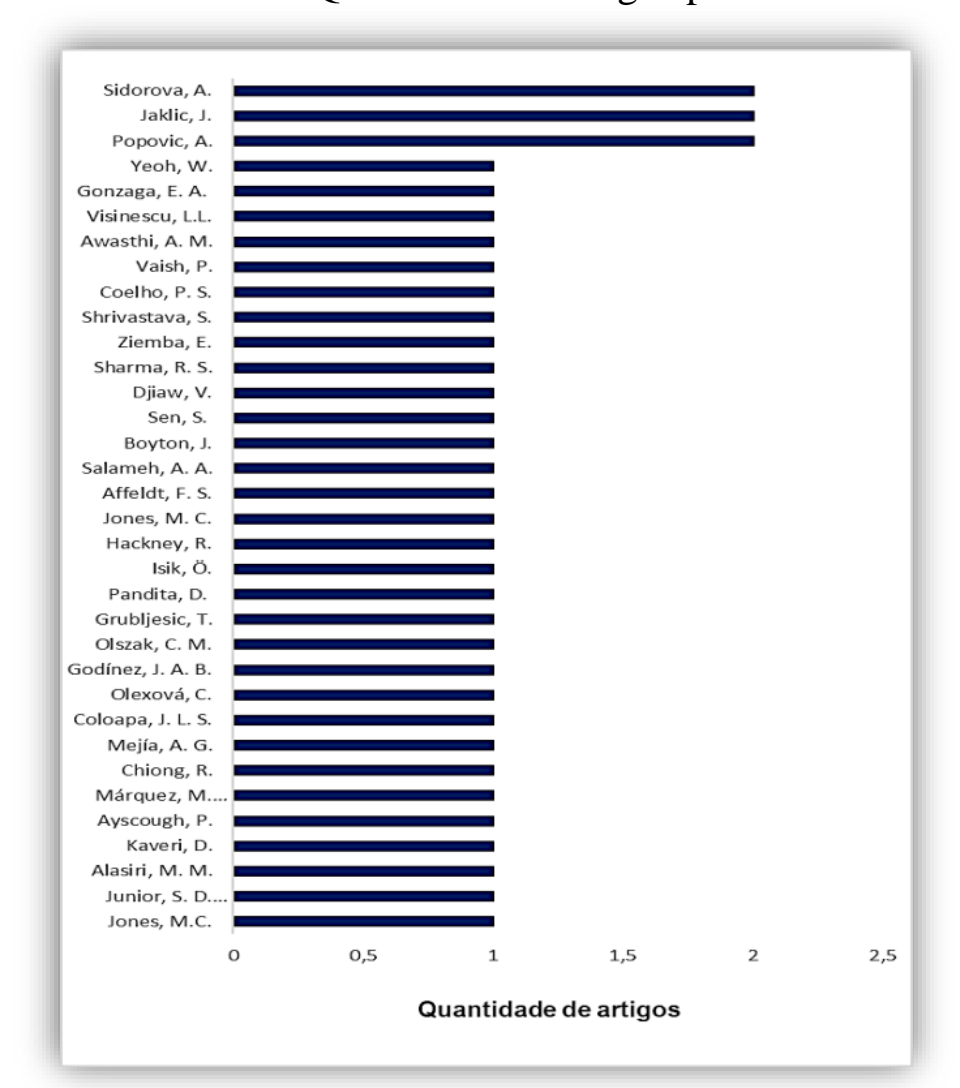

Fonte: Autores (2021). 

decisão: o estado da arte por meio do Proknow. Brazilian Journal of Production Engineering, 8(2), 79-98.

- Ano de publicação dos artigos: relacionando o ano de publicação de cada artigo percebese que o ano de 2020 foi o que mais teve artigo do portfólio publicado, com 3 artigos, conforme Gráfico 5. Lembrando ainda que este número foi limitado ao mês da pesquisa, setembro, ficando subentendido que caso a pesquisa tivesse sido feita levando-se em consideração todo o ano de 2020, a quantidade de artigos tenderia a ser maior.

Gráfico 5. Quantidade de artigos por ano de publicação.

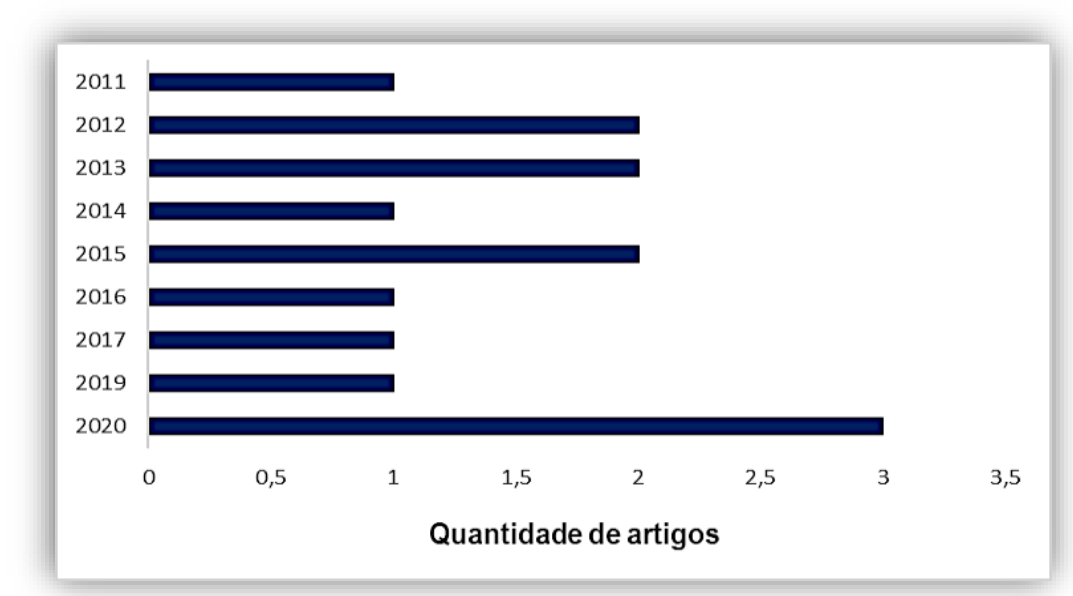

Fonte: Autores (2021).

- Relevância dos autores das referências: compilando a quantidade de vezes que aparece determinado autor nas referências é tido os autores de maior relevância nas referências: são eles Watson, H. J. e Wixom, B. H., conforme a Gráfico 6.

Gráfico 6. Quantidade de vezes da repetição dos autores das referências.

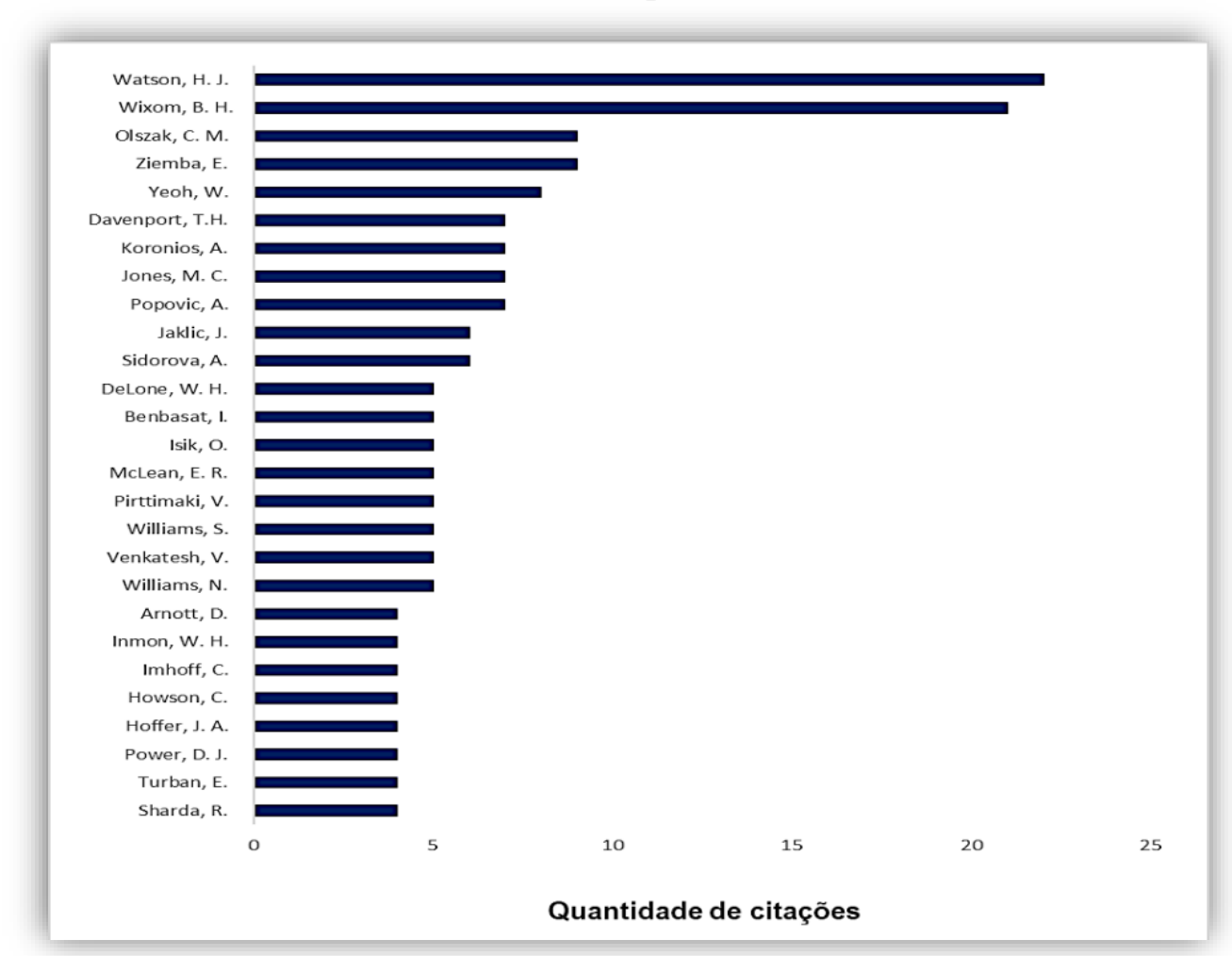

Fonte: Autores (2021). 
Citação (APA): Schinaider, M. A. A., Lee, V. N. T., \& Servare, M. W. J., Jr. (2022). Business intelligence como suporte à tomada de decisão: o estado da arte por meio do Proknow. Brazilian Journal of Production Engineering, 8(2), 79-98.

- Artigos mais citados nas referências: ao analisar a quantidade de vezes que os artigos são citados nas referências percebe-se que os artigos mais citados são: "a empirical investigation of the factors affecting data warehousing sucess" e "critical sucess factors for business intelligence systems", com quatro repetições cada.

Gráfico 7. Quantidade de vezes que um artigo é citado nas referências.

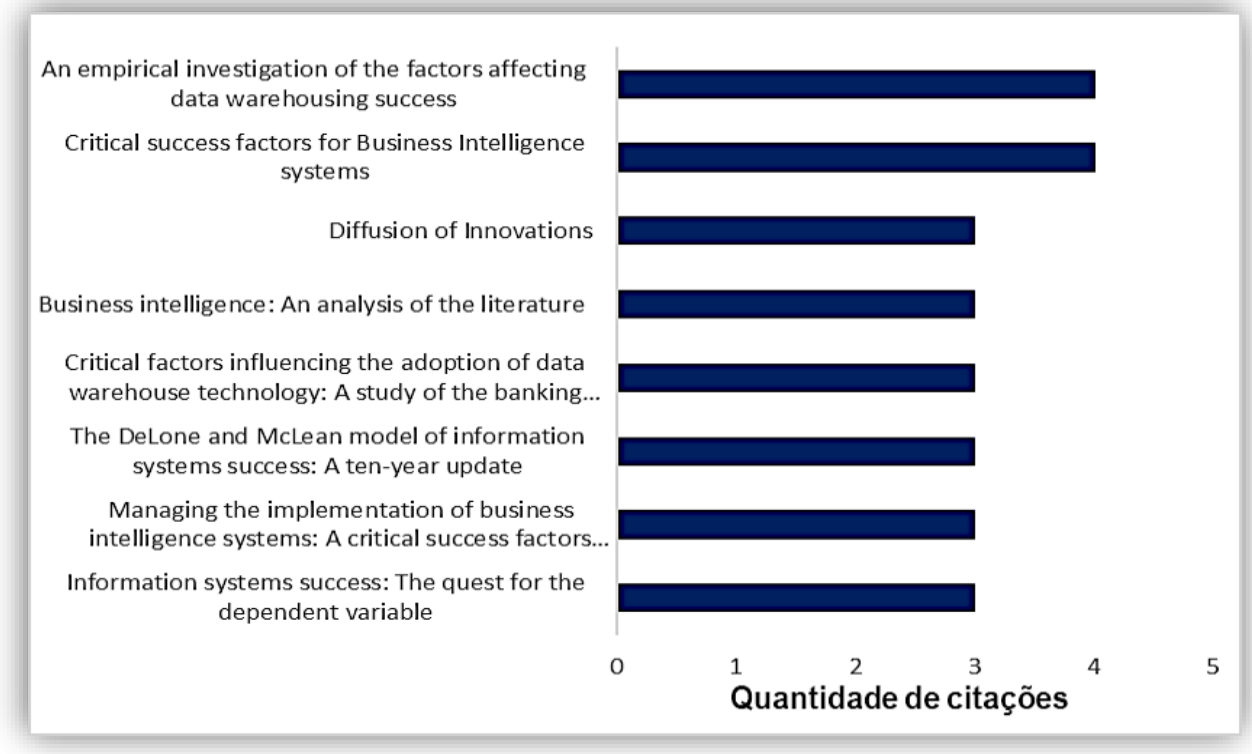

Fonte: Autores (2021).

- Levantamento dos destaques: na matriz a seguir é mostrado o gráfico cujo dois eixos são, número de citação dos artigos no Google Scholar e incidência dos autores dos artigos do portfólio nos artigos de referência. Desta forma, nota-se que cinco artigos são relevantes para o tema, 3 artigos são de destaque, e 3 artigos são de destaque e escrito por autores de destaque.

Gráfico 8. Matriz de artigos e autores de destaque.

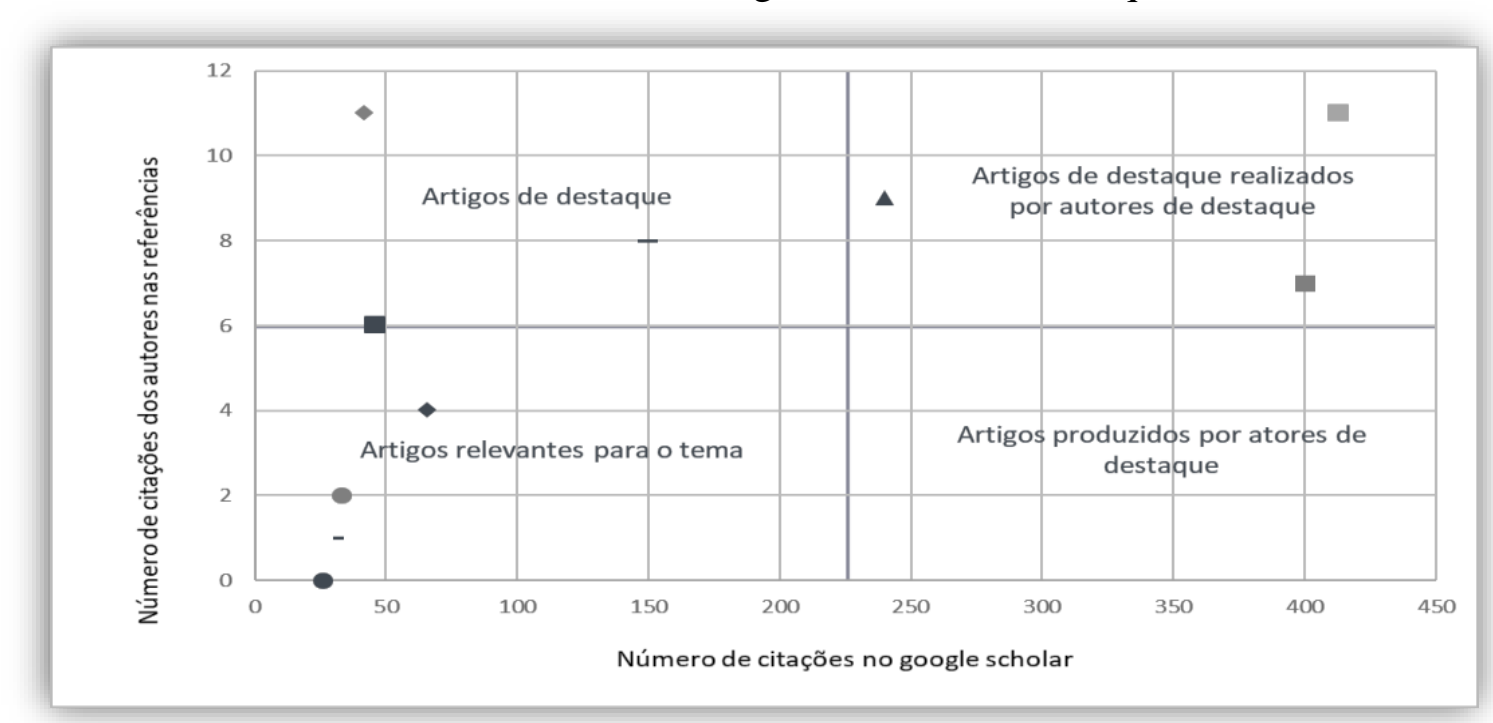

Fonte: Autores (2021).

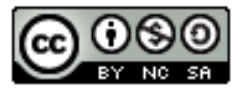


Citação (APA): Schinaider, M. A. A., Lee, V. N. T., \& Servare, M. W. J., Jr. (2022). Business intelligence como suporte à tomada de decisão: o estado da arte por meio do Proknow. Brazilian Journal of Production Engineering, 8(2), 79-98.

Legenda:

\begin{tabular}{|c|c|}
\hline & Information architecture analys is using business intelligence tools based on the information needs of executives \\
\hline & Suboptimal business intelligence implementations: Understanding and addressing the problems \\
\hline & Business intelligence adoption: A case study in the retail chain \\
\hline & Realising the strategicimpact of business intelligence tools \\
\hline & Conceptualization of the business intelligence extended use model \\
\hline & Extending the understanding of critical success factors for implementing business intelligence systems \\
\hline & Improving decision quality: The role of business intelligence \\
\hline & $\begin{array}{l}\text { Critical success factors for implementing business intelligence systems in small and medium enterprises on the } \\
\text { example of upper Silesia, Poland }\end{array}$ \\
\hline & Towards business intelligence systems success: Effects of maturity and culture on analytical decision making \\
\hline & Business intelligence success: The roles of BI capabilities and decision environments \\
\hline
\end{tabular}

\subsection{Análise Sistêmica}

A etapa seguinte foi a análise sistêmica que foi baseada nos objetivos específicos do projeto de pesquisa:

- qual o escopo necessário na implementação de $B I$;

- BI como agente de promoção de competitividade empresarial por meio da tomada de decisão;

- alinhamento entre os resultados obtidos com o uso de $B I$ e os parâmetros referenciais no plano corporativo.

O trabalho de Olexová (2014) propõem etapas para implantação do BI no ambiente corporativo baseado em um estudo de caso em uma empresa do ramo de varejo. $\mathrm{O}$ estudo buscou analisar a implantação de um sistema $B I$ durante determinado período de tempo (cinco meses) levandose em consideração alguns estágios de implantação do sistema: problema inicial; viabilidade do projeto; engenharia de requisitos; projeto; implantação e manutenção. O sistema utilizado foi o SAP BusinessObjects. Após a execução das etapas descritas no quadro 3, os participantes do estudo, integrantes do grupo de gerentes da empresa analisada, perceberam maior agilidade e facilidade no acesso a informações, além da promoção de qualidade operacional. Observou-se também uma boa impressão causada pelo novo sistema, tendo em vista que de nove gerentes entrevistados, seis relataram que o sistema atendia a situação atual da empresa. Por outro lado, como fator preponderante, identificou-se a necessidade de treinamento pela dificuldade de adaptação inicial ao sistema. E por último os participantes relataram melhora da tomada de decisão com o uso do sistema, validando assim a metodologia de implantação aplicada na organização.

\section{Metodologia Aplicada:}

Quadro 3. Metodologia de Implantação de $B I$.

\begin{tabular}{|c|c|}
\hline Problemática & $\begin{array}{c}\text { Identificação da ineficiência do sistema anterior (falta de integração, acesso lento as informações, falta } \\
\text { de dados importantes, previsões incertas). }\end{array}$ \\
\hline Viabilidade & $\begin{array}{c}\text { Formulação do orçamento para investimento na plataforma, implantação e manutenção, bem como o } \\
\text { treinamento dos usuários; análise de custos. }\end{array}$ \\
\hline $\begin{array}{c}\text { Engenharia } \\
\text { de requisitos }\end{array}$ & $\begin{array}{c}\text { Especificação dos indicadores de desempenho (KPIs) necessários ao atendimento às exigências de } \\
\text { informação da organização; } \\
\text { Definição da periodicidade de relatórios; }\end{array}$ \\
\hline Projeto & Definição de soluções técnicas adotadas (OLAP, $D W$, relatórios, etc.). \\
\hline Implantação & $\begin{array}{c}\text { Elaboração de testes no novo sistema, confronto com a ferramenta antiga e aplicação de treinamentos; } \\
\text { Desenvolvimento de dashboards; } \\
\text { Formulação de manuais. }\end{array}$ \\
\hline Manutenção & Localização e correção de erros; e Custos adicionais. \\
\hline
\end{tabular}



decisão: o estado da arte por meio do Proknow. Brazilian Journal of Production Engineering, 8(2), 79-98.

O estudo de Godinez, Coloapa, Márquez, Mejía e Gonzaga (2020) abordou o contexto de pequenas e médias empresas (PMEs) objetivando analisar a implementação eficaz de $B I$ em empresas desses portes. A metodologia utilizada foi revisão bibliográfica e a busca de material para a pesquisa foi feita nas bases: Web of Science, Ebsco e Redalyc. O estudo com base na literatura cita fatores que atrapalham a implementação de $B I$ em empresas como custo do sistema e complexidade. Uma forma encontrada para ajudar na implementação de $B I$ é o treinamento que qualifica os usuários de $B I$ na empresa. Para eficácia na adoção de $B I$ é necessário que a empresa escolha o sistema que combine com sua necessidade e que esteja de acordo com o orçamento previsto. Além disso, o corpo estratégico da empresa deve adotar medidas que favoreçam a cultura do $B I$ dentro da empresa para evitar resistência.

A relevância do treinamento também é relatada no estudo de Awasthi $e$ Pandita (2019), o qual propõem uma análise sobre os aspectos da implementação de $B I$, pela ótica de diminuição de custo proporcionado por fatores como tomada de decisão mais assertiva e levantamento de problemas escondidos, por exemplo. Através de revisão literatura é chegado a importância de a equipe de usuários do $B I$ ser devidamente treinada, pois a utilização de $B I$ pede pessoas capacitadas para isso. Outro ponto levantado é a respeito da necessidade do desenvolvimento de uma cultura favorável ao uso de $B I$ na tomada de decisão, em concordância com o estudo anterior.

O estudo concebido por Grubljesic $e$ Jurij Jaklic (2015), se propõe a investigar a longevidade do uso de $B I$ nas organizações, numa visão além da implementação com o uso forçado, mas na utilização eficaz na tomada de decisão. Para isso o estudo envolveu na sua metodologia, estudo bibliográfico, análise de dois casos práticos e entrevistas com usuários de $B I$ em organizações. A investigação ressalta a importância do suporte do setor de TI para o engajamento do uso das ferramentas, somado a fatores como a idade do usuário e o conhecimento em informática. Além da defesa de que qualidade da informação e do sistema - no que tange a complexidade e acessibilidade - são importantes para a diminuição de resistência no uso, o estudo também defende a relevância da informação, ou seja, quanto menor a lacuna entre o que o sistema oferece e o que os usuários precisam maiores são as chances de sucesso.

Visando o sucesso de implementação de $B I$ ligados fatores críticos de sucesso em organizações, o estudo elaborado por Yeoh $e$ Popovic (2016) aborda fatores em três dimensões: organização, processos e tecnologia:

- Organização: Apoio e comprometimento, e visão clara e caso de negócios bem estabelecido;

- Processos: Competição centrada nos negócios e uma composição de equipe equilibrada, Abordagem interativa de desenvolvimento voltada para negócios e gestão de mudança orientada ao usuário;

- Tecnologia: Estrutura técnica orientada para os negócios, escalonável e flexível e qualidade e integridade de dados sustentáveis.

Dos sete casos estudados identificou-se a necessidade de adaptação dos fatores críticos de sucesso ao contexto da organização, sendo que os fatores ligados a organização, os quais envolvem comprometimento em ajudar e visão clara sobre o negócio, foram os mais importantes para o sucesso na implementação. $\mathrm{O}$ estudo concluiu que os fatores críticos de 

decisão: o estado da arte por meio do Proknow. Brazilian Journal of Production Engineering, 8(2), 79-98.

sucesso promovem a implementação do $B I$ de forma mais assertiva quando implantado na ordem correta. Estes fatores também podem ajudar os líderes a investirem em treinamentos e envolver o funcionário no processo (Yeoh \& Popovic, 2016)

O estudo apresentado por Boyton, Ayscough, Kaveri e Chiong (2015) segue a mesma perspectiva do estudo anterior ao analisar as falhas na implantação de $B I$ e os motivos para tal feito. Com aplicação de uma revisão de literatura identificou-se aspectos ligados as falhas, a mensuração foi tida por meio de quatro fatores: retorno sobre o investimento, medidas de gerenciamento de projetos, satisfação dos usuários e outras medidas avulsas. Conforme constado o insucesso na implantação de $B I$ está relacionado a ausência de fatores críticos de sucesso, conforme as três dimensões, pode-se citar como destaque liderança ineficaz, objetivos pouco claros, falta de planejamento, cultura organizacional que favorece a resistência ao uso de novas ferramentas.

Corroborando nesta linha de pensamento, no estudo realizado por Olszak e Ziemba (2012) é acrescentado outro fator crítico de sucesso importante na dimensão empresa: gestor de business intelligence competente. $\mathrm{O}$ estudo feito com pequenas e médias empresas visou identificar os fatores críticos de sucesso para implementação de $B I$, por meio de entrevistas com os integrantes das empresas, a pesquisa destacou os fatores mais importantes:

- O BI deve estar alinhado a estratégia organizacional abarcando os níveis estratégico, tático e operacional;

- Todos os potenciais usuários do BI devem estar envolvidos no processo de implementação;

- A implementação necessita de pessoal capacitado;

- Necessidade de atenção dispensada pela alta administração ao projeto de implementação;

- Constante desenvolvimento e atualização do sistema;

- Equipe de usuários devidamente capacitada por meio de treinamentos;

- Os custos com ferramentas devem ser vistos como investimento visando manter a frequência do uso.

No estudo realizado por Affeldt $e$ Junior (2013) também por meio de uma pesquisa de campo com um grupo de quatro empresas de diferentes setores ficou evidente a importância do alinhamento da implantação do $B I$ com os objetivos estratégicos. $\mathrm{O}$ foco da pesquisa foi analisar a estruturação de um sistema de informação utilizando da tecnologia de $B I$ e a necessidade de informação dos executivos. Embora o sistema de informação atendesse boa parte das necessidades da empresa pesquisada, elas não atendiam em sua totalidade de acordo com a percepção dos executivos entrevistados. Os fatores que contribuíram para isso foram desconhecimento da capacidade analítica que o sistema de $B I$ pode proporcionar e falta de agilidade na entrega de informações. O que reforça a ideia de uma cultura trabalhada para $\mathrm{o}$ êxito.

No estudo proposto por Vaish, Shrivastava $e$ Sen (2020) é ressaltado a importância da implantação de tecnologias modernas nas empresas visando acesso rápido a informação. Por meio da aplicação de uma revisão de literatura o estudo abordou os conceitos de data mining $(D M)$ e data warehouse (DW), que compõem o framework de $B I$, relacionando com o sistema de apoio a decisão (DSS) visando auxiliar ações estruturadas dentro dos níveis estratégico, tático e operacional na organização. Assim, o estudo buscou verificar se de fato $D M$ e $D W$ 

decisão: o estado da arte por meio do Proknow. Brazilian Journal of Production Engineering, 8(2), 79-98.

contribuem com a eficácia na tomada de decisão dentro da adoção de $B I$ no ambiente corporativo promovendo o diferencial competitivo. $\mathrm{O}$ estudo resultou na efetiva validação das eficiências das estruturas, sendo identificado a redução de gastos, relatórios focados a necessidade real do negócio e promoção de inteligência competitiva. Este último atrelado a entrega de informações de forma mais ágil e qualificadas do que a concorrência. Outro ponto destacado foi a entrega unificada de informações geradas por dados das diversas áreas da empresa, a entrega preditiva disposta pelo $D M$ situa a empresa em um cenário muito mais propício a sua atuação de forma estratégica.

A pesquisa proposta por Isik, Jones $e$ Sidorova (2013) visou apurar a relação entre o uso das ferramentas de $B I$ e seu êxito em diferentes contextos de gestão. Sucesso no trabalho foi entendido como a boa visão que os usuários possuem sobre a colaboração do $B I$ e atrelado a fatores como lucratividade e efetividade corporativa. É pressuposto que o insucesso de $B I$ pode estar ligado a fatores como desalinhamento entre os objetivos e a implantação do sistema. As empresas tem investido mais em quantidade, do que qualidade de dados. Os autores acreditam que um dos quesitos mais relevantes no sucesso de $B I$ é a flexibilização do sistema, ou seja, a capacidade do sistema adapta-se as oscilações nos processos da empresa. Outro ponto consiste na possibilidade dos sistemas de $B I$ permitirem a empresa tomar decisão frente a cenários incertos. Diante desses fatores o estudo elencou hipóteses, em seguida prosseguiu-se com verificação das hipóteses traçadas por meio de uma metodologia envolvendo entrevista com gerentes de negócios de setores variados. O estudo conclui que a qualidade de acesso, integração e flexibilidade dos sistemas contribuem para a eficácia do $B I$, apesar disso, as organizações precisam estar atentas as formas de acessos dispostas para seus usuários dos sistemas. Outro ponto constatado foi a competitividade atrelado aos ambientes de decisão, o importante é que o usuário sinta confiança em usar $B I$ em qualquer sistema. Estes são os fatores para alcance da vantagem competitiva.

O projeto de pesquisa elaborado por Alasiri $e$ Salameh (2020), investiga os impactos do BI e do Sistema de apoio a decisão para a empresa, a forma de implementação e os benefícios percebidos. Foi realizada pesquisa bibliográfico com portfólio selecionado criteriosamente. $\mathrm{O}$ estudo comprova a eficácia de Business intelligence no suporte a tomada de decisão possibilitando percepções verdadeiras em várias áreas do negócio. Mas ainda assim foram identificados fatores que atrapalham as organizações atingirem o objetivo com BI. Tais como: falta de gerenciamento dos dados, com a probabilidade de uso de dados falso; a necessidade de autorização dos dados, limitação do orçamento; falta de especialização, que pode levar a análises errôneas e consequente comprometimento da tomada de decisão assertiva.

O estudo proposto por Visinescu, Jones $e$ Sidorova (2015) analisou a percepção da qualidade de decisão para vantagem competitiva, de um grupo seleto composto de trabalhadores e estudantes que já observaram o uso do BI. Foram aplicados medidores encontrados na literatura para validação de cinco hipóteses formuladas que envolvem três fatores mesclado entre eles, nível do uso do sistema, complexidade do local de aplicação e qualidade da informação. Assim, quatro hipóteses foram aceitas e uma foi contestada. Em suma, também se identificou que mesmo que com alto nível de uso de $B I$ na empresa, informações de baixa qualidade leva a percepção de baixa qualidade na tomada de decisão pelos usuários, sendo que neste cenário o 

decisão: o estado da arte por meio do Proknow. Brazilian Journal of Production Engineering, 8(2), 79-98.

alto nível do uso se justifica devida a obrigatoriedade imposta pela empresa. O uso de $B I$ promove uma tomada de decisão mais assertiva quando a qualidade da informação provida pelos sistemas é alta, por isso as empresas necessitam checar a qualidade da informação passada para seus usuários. Além disso, foi constatado que as informações advindas dos sistemas de $B I$ são taxadas como melhores e causam mais impacto nos usuários. Em relação a complexidade do ambiente foi percebido que a percepção da tomada de decisão de maior qualidade está relacionada a ambientes de menor complexidade, este fato é relacionado à capacidade limitada de processamento de informações pelos tomadores de decisão, quanto mais fatores envolvidos mais complexo será tomar a decisão, mesmo com sistemas de $B I$.

O estudo elaborado por Popovic, Hackney, Coelho $e$ Jaklic (2012) segue nesta linha, segundo os autores a qualidade da informação é afetada pela maturidade da implementação de $B I$ a se comportar de forma concomitante, a qualidade envolve dois fatores destacados pelos autores, qualidade do conteúdo e do acesso, em concordância com outros autores citados anteriormente. Contudo as empresas costumam dar menos importância para o fator qualidade do conteúdo devido, em geral, a implementação ser direcionada ao viés tecnológico e não olhado pelas necessidades de informação dos usuários. O estudo defende que para alcançar o diferencial competitivo o fator qualidade do conteúdo de informação é mais importante que o fator de acesso, isso porque informações desencontradas com os objetivos estratégicos da empresa podem continuar levando a organização a fazer decisões menos assertivas, o que acarretará na insatisfação e consequente abandono cada vez maior do uso dos sistemas. Em resumo, a maior utilização de $B I$ no ambiente corporativo não promove sucesso na gestão, se as informações não estiverem ajustadas ao seu propósito. É primordial definir metas, monitorá-las e corrigi-las sempre que necessário.

Visando contribuir com a melhoria da performance de gestão de empresas, o estudo feito por Sharma $e$ Djiaw (2011) analisou o uso do balanced scorecard (BSC), ferramenta que colabora com a execução do plano estratégico de empresas por meio de mensurações de desempenho (Geishecker \& Rayner apud Sharma \& Djiaw, 2001), em complementação ao BI averiguando a eficácia na gestão de conhecimento das empresas para atingir vantagem competitiva. No estudo de campo feito em uma empresa foi constado que a falta de confiança nas ferramentas levava aos colaboradores ao uso do $B I$ de forma não eficiente. Sugeriu-se que a organização deva estabelecer um plano estratégico que atenda às necessidades dela, neste plano devem incluir orientações sobre como utilizar as ferramentas de $B I$ para atingir a estratégia da empresa e alinhe a execução com a estratégia. Conclui-se que a gestão estratégica e o uso de ferramentas de $B I$ em conjunto promovem maior competitividade a empresa, além disso a empresa deve promover o compartilhamento de conhecimento entre os colaboradores.

\section{CONSIDERAÇÕES FINAIS}

O presente trabalho abordou uma pesquisa sobre a relevância do business intelligence no ambiente de tomada de decisão. Para isso o estudo adotou a metodologia de revisão de literatura, proknow- $C$ visando a formação do portfólio bibliográfico, o qual foi analisado sobre a visão de três aspectos: relevância acadêmica do assunto, implementação no ambiente corporativo e promoção de competitividade empresarial por meio da tomada de decisão.

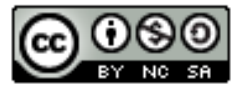



decisão: o estado da arte por meio do Proknow. Brazilian Journal of Production Engineering, 8(2), 79-98.

Em primeiro lugar, foram analisados os artigos sobre implementação do $B I$. Um fator predominante para o sucesso bastante citado diz respeito ao treinamento dos usuários, pois a eficácia da implementação exige corpo técnico preparado. Este quesito também está ligado a promoção de uma cultura menos resistente ao uso de novas tecnologias dentro das empresas, outro fator apontado como crítico ao sucesso da implantação. Assim, a metodologia base proposta e validada para implantação consiste em: problemática, validação, requisitos, projeto, implementação e manutenção do sistema de $B I$.

Ainda sobre os sistemas de $B I$, as organizações devem avaliar o sistema mais adequado as suas necessidades de informação a respeitar o orçamento estipulado dentro da etapa de validação do projeto, pois o alto custo, além da complexidade dos sistemas, são fatores que atrapalham a implantação. É esperado que o investimento usado na implementação de ferramentas de $B I$ retorne com as vantagens competitivas atreladas ao seu uso.

Os fatores críticos de sucesso ligados a organização foram os mais citados dentro da coletânea estudada como importantes para o sucesso da adoção do BI no ambiente corporativo. Estes fatores estão ligados a uma visão clara sobre o negócio e o comprometimento da liderança junto a sua equipe buscando auxiliá-los no que for preciso para a efetividade da implantação. Pois a falta de capacitação do gestor é um dos aspectos que podem levar a implantação ao insucesso. Outros fatores são falta de planejamento e falta de envolvimento dos usuários de informações.

Sobre a visão do diferencial competitivo foi analisada a influência na tomada de decisão. $\mathrm{O}$ diferencial em relação a concorrência consiste na leitura de informações focadas no negócio, ágeis e de qualidade, possibilitando ainda a análise preditiva sobre os diversos ambientes que rodeiam a empresa. Em geral para ser competitivo o sistema implantado deve ser flexível, fácil de integração entre a forma de uso de cada usuário (aplicativo, sistema web, software, etc.).

Foi citado em mais de um artigo que a qualidade do conteúdo da informação é mais importante, se comparado o nível de uso, todavia é comum dentro das organizações a usabilidade ser maior do que a qualidade da informação. Isso porque a implantação costuma ser voltada para tecnologia, deixando de lado o fator humano que proporciona entender a real necessidade da adoção do BI. Mesmo assim, grande parte dos usuários utilizam as ferramentas por imposição das empresas, que por sua vez não se preocupam em trabalhar a informação gerada.

O sucesso competitivo também é atrelado ao ambiente de decisão que o usuário está inserido. Quanto mais complexo for o ambiente e mais variáveis atingirem o indivíduo, maior a dificuldade de acesso à informação ideal para tomada de decisão. Em qualquer situação, a organização necessita preparar um meio seguro para o uso de informações de forma estratégica. Uma ação apontada pela literatura para isso é a adoção de orientações de uso dos sistemas dentro do plano estratégico. Conclui-se que a gestão estratégica e o uso de ferramentas de $B I$ em conjunto promovem maior competitividade para a empresa, além disso, deve-se promover o compartilhamento de conhecimento entre os colaboradores.

Desta forma, a pesquisa atingiu o seu objetivo de elucidar os impactos do $B I$ nas empresas como provedor do manuseio estratégico de informações baseado nas métricas relacionadas ao planejamento estratégico para auxílio na tomada de decisão. É sugerido que para pesquisas futuras a partir do conhecimento do portfólio bibliográfico, da identificação das características 
Citação (APA): Schinaider, M. A. A., Lee, V. N. T., \& Servare, M. W. J., Jr. (2022). Business intelligence como suporte à tomada de decisão: o estado da arte por meio do Proknow. Brazilian Journal of Production Engineering, 8(2), 79-98.

desse portfólio e da análise sistêmica, a continuidade da pesquisa com o desenvolvimento da etapa de identificação de oportunidades de pesquisa por meio do ProKnow-C.

\section{REFERENCIAS}

Abusweilem, M. A. \& Abualoush, S. (2019). The impact of knowledge management process and business intelligence on organizational performance. Growing Science, 2143-2156. http://doi.org/10.5267/j.msl.2019.6.020.

Affeldt, F. S. \& Junior, S. D. da S., Jr. (2013). Information architecture analysis using business intelligence tools based on the information needs of executives. JISTEM - Journal of Information Systems and Technology Management, 10(2), 251-270. https://doi.org/10.4301/S1807-17752013000200004.

Afonso, M. H. F., Souza, J. V. de, Ensslin, S. R., \& Ensslin, L. (2011). Como construir conhecimento sobre o tema de pesquisa? aplicação do processo proknow-c na busca de literatura sobre avaliação do desenvolvimento sustentável. Revista de Gestão Social e Ambiental, 5(2), 47-62. https://doi.org/10.24857/rgsa.v5i2.424.

Agiu, D., Mateescu, V., \& Muntean, I. (2014). Business intelligence overview. Database system Journal, 5(3), 23-36. Recuperado de http://dbjournal.ro/archive/17/17_3.pdf

Alasiri, M. M. \& Salameh, A. A. (2020). The impact of business intelligence (BI) and decision support systems (DSS): exploratory study, International Journal of Management (IJM), 11(5), 1001-1016. https://doi.org/ 10.34218/IJM.11.5.2020.092

Armstrong, P. (Ed), (2019). Dominando as tecnologias disruptivas: aprenda a compreender, avaliar e tomar melhores decisões que possa impactar o seu negócio (1a ed. p. 19-20). São Paulo: Autêntica Business.

Awasthi, A. M. \& Pandita, D. (2019). Role of business intelligence and analytics: analysis of data driven decision. International Journal of Innovate Technology and Exploring Engineering (IJITEE), 8(12), 1506-1510. https://doi.org/10.35940/ijitee.13101.1081219.

Boyton, J., Ayscough, P., Kaveri, D., \& Chiong, R. (2015). Suboptimal business intelligence implementations: understanding and addressing the problems. Journal of Systems and Information Technology, 17(3), 307-320. https://doi.org/10.1108/JSIT-03-2015-0023.

Delen, D., Turban, E., \& Sharda, R. (Ed). (2015). Business intelligence and analytics: systems for decision support (10th ed). Oklahoma: Pearson.

Ensslin, L., Graziano, L. A. G., Dutra, A., \& Dezem, V. (2017). Construção de conhecimento sobre o tema avaliação de desempenho da comunicação em órgãos públicos: uma análise da literatura internacional. Revista Ibero-Americana de Estrategia - RIAE, 16(3), 111-129. https://doi.org/10.5585/riae.v16i3.2519.

Ensslin, S. R.; Ensslin, L., Yamakawa, E. K., Nagaoka, M. da P. T., Aoki, A. R., \& Siebert, L. C. (2014). Processo estruturado de revisão da literatura e análise bibliométrica sobre avaliação de desempenho de processos de implementação de eficiência energética. Revista Brasileira de Energia, 20(1), 21-50. Recuperado de https://sbpe.org.br/index.php/rbe/article/view/319.

Fontana, L. P. B. (2020). Modelo multicritério construtivista para apoiar a gestão organizacional em uma empresa concessionária de serviços de saneamento no Brasil. (Dissertação de mestrado). Universidade do Sul de Santa Catarina, Florianópolis, Santa Catarina, Brasil.

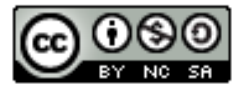


Citação (APA): Schinaider, M. A. A., Lee, V. N. T., \& Servare, M. W. J., Jr. (2022). Business intelligence como suporte à tomada de decisão: o estado da arte por meio do Proknow. Brazilian Journal of Production Engineering, 8(2), 79-98.

Godínez, J. A. B., Coloapa, J. L. S., Márquez, M. S. U., Mejía, A. G., \& Gonzaga, E. A. (2020). Identifying the main factors involved in business intelligence implementation in SMEs. Bulletin of Electrical Engineering and Informaties. 9(1), 304-310. https://doi.org/10.11591/eei.v9i1.1459.

Grubljesic, T. \& Jaklic, J. (2015). Conceptualization of the business intelligence extended use model. Journal of Computer Information Systems, 55(3), 72-82. https://doi.org/10.1080/08874417.2015.11645774.

Isik, O., Jones, M. C., \& Sidorova, A. (2013). Business intelligence success: the roles of BI capabilities and decision environments. Information \& Management, 50(1), 13-23. https://doi.org/10.1016/j.im.2012.12.001.

Lacerda, R. T. de O., Ensslin, L., \& Ensslin, S. R. (2012). Uma análise bibliométrica da literatura sobre estratégia e avaliação de desempenho. Gestão \& Produção, 19(1), 59-78. https://doi.org/10.1590/S0104-530X2012000100005.

Lizot, M. Junior, P. P. de A., Magacho, C. S., Bortoluzzi, S. C., \& Viero, A. (2016). Avaliação de desempenho na gestão da produção: análise bibliométrica e sistêmica da literatura internacional. Revistas Gestão Industrial. 12(3), 65-84. https://doi.org/10.3895/gi.v12n3.4377.

Nedelcu, B. (2013), Business intelligence systems. Database system Journal, 4(4), 12-20. Recuperado de http://www.dbjournal.ro/archive/14/14.pdf\#page=13

Olexová, C. (2014). Business intelligence adoption: a case study in the retail chain. WSEAS Transactions on Business and Economics, 11(1), 95-106. Recuperado de http://www.wseas.us/journal/pdf/economics/2014/a185707-163.

Olszak, C. M. \& Ziemba, E. (2012). Critical sucess factors for implementing business intelligence systems in small and medium enterprises on the example of upper Silesia, Poland. Interdisciplinary Journal of Information, Knowledge, and Management, 7, 130-150. https://doi.org/10.28945/1584.

Popovic, A., Hackney, R., Coelho, P. S., \& Jaklic, J. (2012). Towards business intelligence systems success: effects of maturity and culture on analytical decision making. Decision Support Systems, 54(1), 729-739. https://doi.org/10.1016/j.dss.2012.08.017.

Reina, D. R. M., Ensslin, S. R., Ensslin, L., \& Reina, D. (2014). Seleção e análise do perfil da produção científica sobre $\mathrm{o}$ tema seleção de projetos. REGE, 21(1), 3-25. https://doi.org/10.5700/rege516.

Rogers D. L. (Ed). (2017). Transformação digital: repensando o seu negócio para era digital (2nd ed. p. 121-130). São Paulo: Autêntica Business.

Santos, A. I. dos, Schenatto, F. J. A., \& Oliveira, G. A. (2017). Metodologia proknow-C para construir o conhecimento acerca de previsão de demanda utilizando séries temporais. Ponta Grossa/PR. VII Congresso Brasileiro de Engenharia de Produção.

Schwab, K. (Ed). (2016). A quarta revolução industrial (1a ed. p. 23). São Paulo: Edipro.

Sharma, R. S. \& Djiaw, V. (2011). Realising the strategic impact of business intelligence tools. VINE, 41(2), 113-131. https://doi.org/10.1108/03055721111134772.

Silva, L. H. B. da, Bezerra, J. C. C., Rios, F. F. S., \& Amorim, F. A. (2019). Desenvolvimento de dashboards interativos utilizando ferramentas de business intelligence no ms excel para auxilio na tomada de decisão empresarial. Revista Expressão Católica, 7(1), 27-38. http://dx.doi.org/10.25190/rec.v7i1.2129.

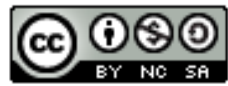



decisão: o estado da arte por meio do Proknow. Brazilian Journal of Production Engineering, 8(2), 79-98.

Vaish, P., Shrivastava, S., \& Sen, S. (2020). Business intelligence: escalation of data warehousing and data mining for effective decision making. International Jounal of Advanced Science and Technology, 29(5), 1377-1388. Recuperado de http://sersc.org/journals/index.php/IJAST/article/view/8178.

Vaz, C. R., Tasca, J. E., Ensslin, L., Ensslin, S. R., \& Selig, P. M. (2012). Avaliação de desempenho na gestão estratégica organizacional: seleção de um referencial teórico de pesquisa e análise bibliométrica. Revista Gestão Industrial. 8(4), 121-153. https://doi.org/10.3895/S1808-04482012000400008.

Vergara, S. C. (Ed), (2016). Projetos e relatórios de pesquisa em administração (16th ed.). São Paulo: Editora Atlas.

Vieira, E. L., Bortoluzzi, S. C., Costa, S. E. G. da., \& Lima, E. P. de. (2017). Processo estruturado de revisão da literatura e análise bibliométrica sobre avaliação do nível de maturidade das empresas na utilização de ferramentas lean manufacturing. Revista LatinoAmericana de Inovação e Engenharia de Produção, 5(7), 64-79. http://dx.doi.org/10.5380/relainep.v5i7.55173.

Visinescu, L. L., Jones, M. C., \& Sidorova, A. (2015). Improving decision quality: the role of business intelligence. Journal of Computer Information Systems, 57(1), 58-66. https://doi.org/10.1080/08874417.2016.1181494.

Yeoh, W. \& Popovic, A. (2016). Extending the understanding of critical success factors for implementing business intelligence systems. Journal of the Association for Information Science and Technology, 67(1), 134-147. https://doi.org/10.1002/asi.23366.

Zanella, L. C. H. (2009). Metodologia de Estudo e Pesquisa em Administração. UFSC, Florianópolis, SC, Brasil. 